\section{Tumours of Retrorectal Space: A Rare Entity Scarcely Known by General Surgeons}

\section{Abstract}

Neoplasms occurring in the retrorectal space are an uncommon condition with heterogeneous aetiology. Their incidence is estimated in the range 1: 40,000 to 1: 63,000 admissions. Consequently, most surgeons are not familiar with this condition and diagnosis is a challenge. Some authors have reported an increased incidence in female gender. According to Lovelady and Dockerty, they are classified into congenital (two-thirds), neurogenic, osseous and miscellaneous. Most of patients are asymptomatic and when symptoms appear they are non-specific and diffuse: back pain, constipation, infection, chronic perineal pain, dimpling of the skin, gynaecological complaints etc. An adequate diagnosis is crucial to reach a proper treatment. Computed tomography and magnetic resonance imagining are useful tools. Surgery is considered the gold standard treatment. Furthermore, a multidisciplinary approach is required. It must involve colorectal surgeons, radiologists, pathologists, neurologists and traumatologists. We report our experience in these lesions for a 10 year period in an attempt to reach conclusions about the management of this unusual entity.

Keywords: Retrorectal; Presacral; Hamartoma, Tumours; Congenital; Rectum

\section{AM Minaya-Bravo, E Ovejero Merino and M Diaz-Alonso}

1 Colorectal Surgeon, Principe de Asturias Hospital, Carretera de Alcala-Meco, Madrid, Spain

2 Resident of General Surgery. Principe de Asturias Hospital, Carretera de AlcalaMeco, Madrid, Spain

Corresponding author: AM Minaya-Bravo

戸 ana-minaya@hotmail.com

Ana Maria Minaya Bravo

Colorectal Surgeon, Principe de Asturias Hospital, Carretera de Alcala-Meco, Madrid, Spain

Tel: 0034918878100

Citation:AM Minaya-Bravo, Merino EO, Alonso MD. Tumours of Retrorectal Space: A Rare Entity Scarcely Known by General Surgeons. J Univer Surg. 2017, 5:1.

\section{Introduction}

Rectrorectal tumours are a extremely rare entity. According to Jao et al. [1] these tumours account for 1 in 40,000 hospital admissions [1-3]. Some authors have reported a rise of female gender cases (ratio 3:1) with a peak at middle age [4-7]. In addition, malignant processes seem to be more frequent in women than in men $[3,4]$. Moreover, malignancy is also associated to solid aspect and paediatric population. Most of surgeons will face only one or two cases along their career. As a result, misdiagnosis and delayed treatment are frequent. Therefore, a proper anatomical knowledge of retrorectal space is crucial to achieve a comprehensive understanding and an adequate treatment. The vast majority of these lesions are benign and congenital. However, there are a small percentage of malignant tumours that make surgery highly recommended when conditions of the patient are optimal. Furthermore, surgery provides not only treatment but also definitive histopathological diagnosis [4,7-11]. Two essential tools are magnetic resonance imagining (MRI) and rigid rectoscopy, both of them allow to plan an adequate surgical procedure. Surgical procedure depends on nature of lesion and location. Digital rectal examination and rigid rectoscopy will allow to locate the lesion and, subsequently, to perform a multidisciplinary approach. Lesions placed above S3 need an abdominal incision and those below S3 can be approached through via posterior (Kraske procedure) [4-6,11].

\section{Method}

We reviewed the cases of retrorectal tumours admitted in our hospital from 2000 to 2015 . We only treated four patients. It confirms the rarity of this entity. From these three patients only three underwent surgery and one of them rejected surgery and is still under surveillance. All of them were transferred to Colorectal Department and treated by senior surgeons.

\section{Cases}

\section{Case 1}

A 42 year old woman was admitted to our Department with perianal pain. She did not have relevant medical history. 
She complained perianal pain irradiated to the sacrum and constipation for the last 3 months. Digital rectum examination revealed a smooth mass, compressing posterior wall of rectum from outside, at $4 \mathrm{~cm}$ from anal verge. No fistulous tracts, postanal skin dimple or other external signs were observed.

MRI showed a $3 \times 4 \mathrm{~cm}$ size presacral mass with cystic aspect and septa inside, suggesting hamartoma. It extended from S3 level to coccygeal region. Invasion of adjacent structures were not demonstrated. Computed tomography (CT) confirmed findings of MRI. Colonoscopy revealed an image of extrinsic compression on the posterior rectal wall at $4 \mathrm{~cm}$ from anal verge. The patients underwent surgery. Kraske procedure was chosen based on the location of the lesion. A parasacral incision was performed. Anococcygeal ligament was excised, gluteus maximus muscles separated laterally and coccix excised. A circumferential excision was performed because of the nature of the lesion, and the lesion was separated from the mesorectum easily (Figure 1). Frozen biopsy reported cystic hamartoma of $4 \mathrm{~cm}$ diameter, multilocular with transitional lining and disorganized smooth muscle. The patient was discharged after 5 days. After 8 years follow-up, no recurrence has been demonstrated.

\section{Case 2}

A 62 year old woman was presented to the Colorectal Department with a retrorectal tumour as an incidental finding on pelvis CT requested by traumatologist. She had unremarkable past medical history. She referred lower back pain in the last months. She did not report any other symptom. Digital rectum examination showed a submucosal lesion placed in the posterior rectal wall. CT showed a structure that sized $3 \mathrm{~cm}$ diameter across with unilocular cystic aspect with thin wall. A MRI multilayer study revealed a $3 \mathrm{~cm} \times 2.5 \mathrm{~cm} \times 2 \mathrm{~cm}$ cysts in the retrorectal space at the level S2-S3, with a well -defined wall, high protein content and hypointense in T2. No invasion of adjacent structures was demonstrated (Figure 2). Colonoscopy was performed and it illustrated an image of extraluminal compression of the rectum on posterior wall, at $5 \mathrm{~cm}$ from anal verge. Tumoral markers were normal. The patients underwent surgery. Kraske procedure was performed with excision of coccyx. Circumferential excision was carried out. Frozen section exam revealed a $3.5 \mathrm{~cm}$ dermoid cyst with keratin and fibronecrotic material inside. She was discharged after 3 days. After 3 year follow-up she remains free of disease.

\section{Case 3}

A 39 year old man was admitted to our department with rectal tenesmo for two months. He had irrelevant past medical history. On digital rectum examination, no mass was palpable. There was no evidence of perianal lesions. Colonoscopy demonstrated an extrinsic, well-defined presacral mass compressing the rectum, at $7 \mathrm{~cm}$ of anal verge. MRI illustrated a $3 \mathrm{~cm}$ size cystic lesion in the retrorectal space at $\$ 4$ level. Multilocular, with septa and fluid level. It did not invade surrounding structures. It was reported as hamartoma (Figure 3). In this case, an abdominal approach was considered. The patient was informed of his disease and advised to undergo surgery to provide definitive diagnosis and alleviate his symptoms. However, the patient rejected surgery and decided to continue under surveillance. Biopsy was not considered because of high risk of infection. A pelvis MRI is performed every 6 months. After follow-up of 4 years, the lesion remained stable, no new lesions have appeared and patient is asymptomatic.
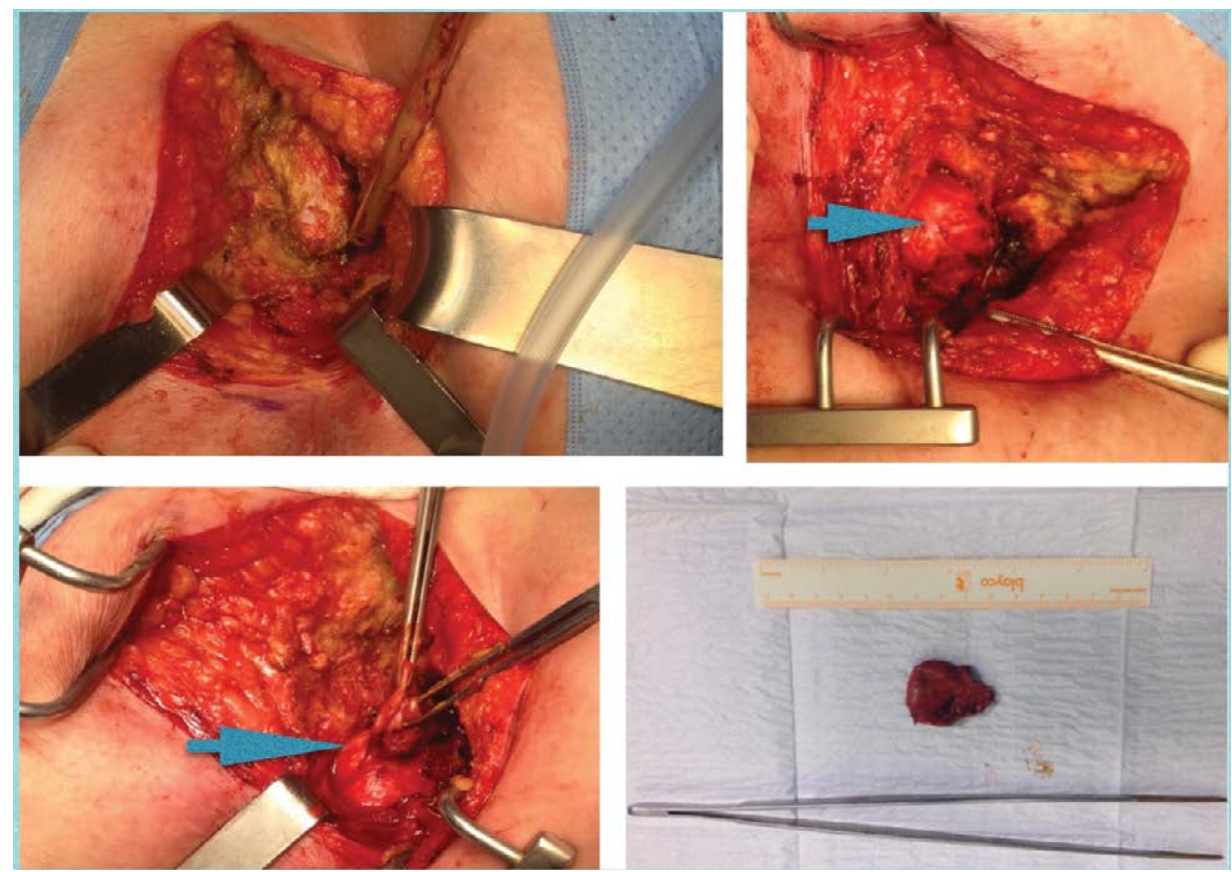

Figure 1 Kraske procedure is illustrated. A circumferential excision was performed to remove a harmatoma tumour. 

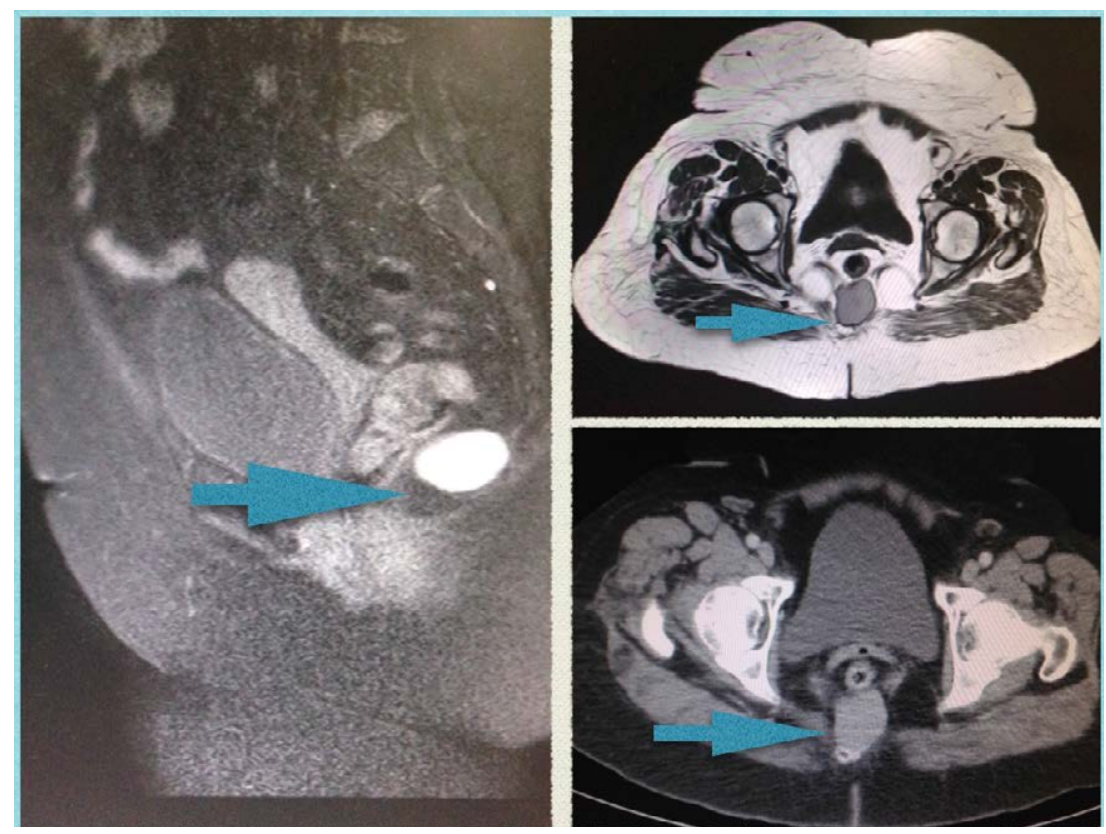

Figure 2 CT and MRI showed a dermoid cyst with unilocular aspect.
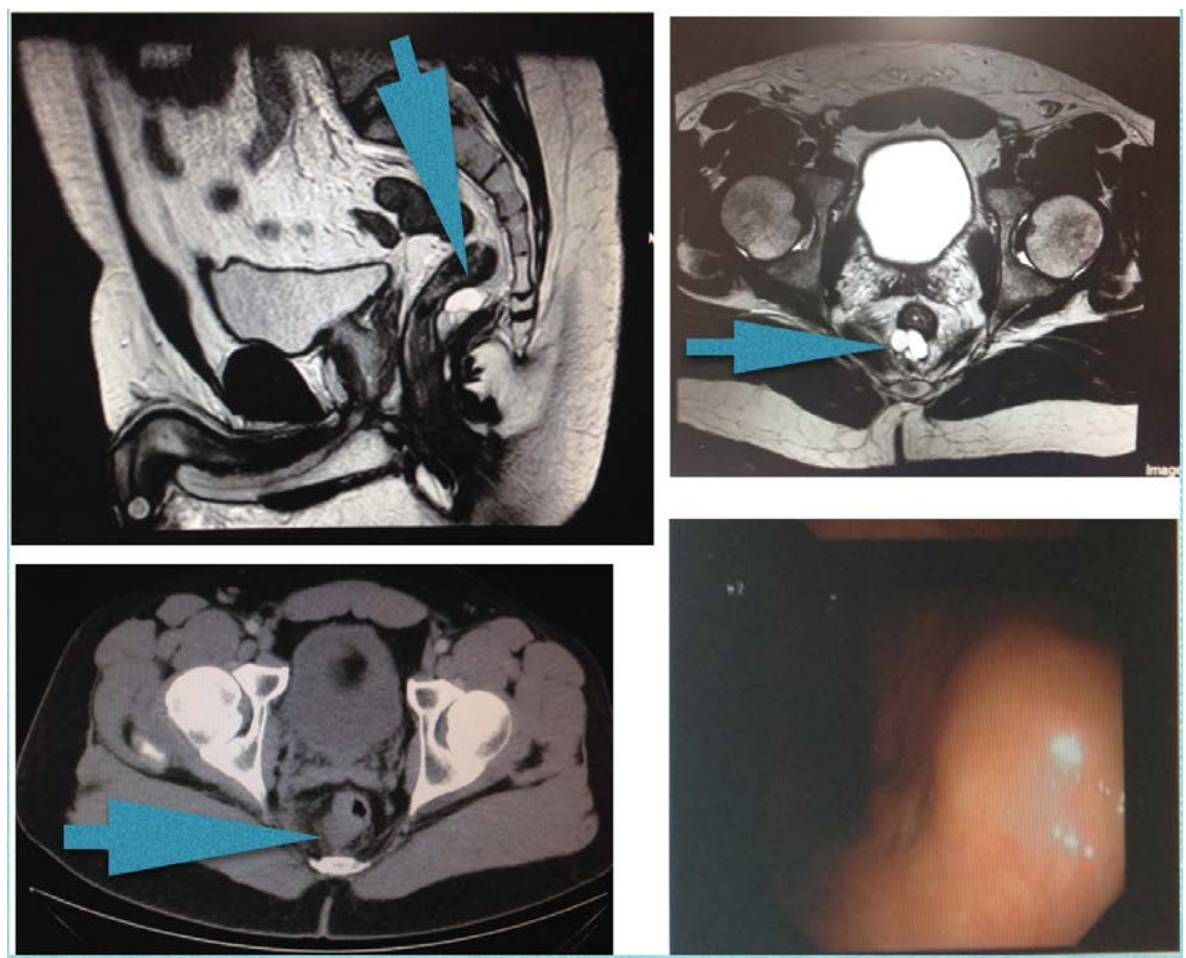

Figure 3 Harmatoma tumour was revealed in CT and MRI as a multilocular cyst with septa and liquid level. Colonoscopy showed an extraluminal compression.

\section{Case 4}

A 65 year old woman with a retrorectal tumour in $\mathrm{CT}$ was presented to our department; she had suffered from pulmonary embolism six months ago. She complained of coxalgy. On digital examination, at the tip of the examining finger, a smooth mass was palpable in the posterior wall of rectum. CT and MRI revealed a $3.5 \mathrm{~cm}$ cyst lesion, at $5 \mathrm{~cm}$ of anal margin. She underwent surgery. Kraske procedure was performed as described previously. A circumferential excision realized and a 
small fibrous tract connected to rectum evidenced and sutured (Figure 4) Postoperative period was uneventful, and the patient discharged after 3 days. After 1 year follow-up, no recurrence has been demonstrated.

\section{Discussion}

An accurate anatomical description is necessary to understand these tumours. The retrorectal space is bounded posteriorly by parietal presacral fascia and sacrum, anteriorly by rectum and mesorectum covered by a fine visceral fascia, superiorly by peritoneal reflection, inferiorly by levator ani muscle and laterally by iliac vessels, sacral nerves roots, lateral stalks of rectum and ureters (Figure 5A) $[2,5,7,12]$. The rectosacral fascia (Waldeyer's fascia) divides the rectrorectal space into superior and inferior compartment. This fascia originates from the parietal presacral fascia at the level of S2-S4 and passes caudally to reach the rectum at $3 \mathrm{~cm}$ to $5 \mathrm{~cm}$ above anorectal junction (Figure 5B) [2,12]. In this space occurs a series of events that produces the differentiation of totipotent stem cells into three germ cell layers: ectoderm, mesoderm and endoderm. Eventually, a great variety of tissue is stemmed from these three layers: connective tissue, neural elements and bone. According to this, tumours originated from this space constitute a varied group [2,5]. Several classifications have been used. Firstly, in 1949, Lovelady and Dockedty classified them into: congenital, inflammatory, neurogenic, osseous and miscellaneous [2,5,6,8,9]. Afterwards, in 1975, Uhlig and Johnson noted 63 patients treated over 30 year period in Oregon area and classified them using prototype of Lovelady and Dockedty classification [2,11]. Finally, Dozois et al. subcategorised these tumours into malignant and benign processes. In 2003, LevChelouche et al. added other subclassification: congenital versus acquired, resulting into four categories; malignant, benign, congenital and acquired [2]. Most of the lesions are congenital, benign and more common in women [4,5]. Classification according to Lovelady and Docketdy includes $[2,5,6,8,9]$.

\section{Congenital lesions}

Developmental cysts: Account for $60 \%$ of all congenital lesions. Complications are not rare and include infection, abscess, fistula and in some cases malignancy.

They are divided into:

*Epidermoid and dermoid cysts: benign unilocular lesions. Both of them may be associated to sinus or

postanal dimple.

*Enterogenous cyst: Also known as duplication cysts of rectum. They are featured by mucosal lining, muscular wall with mesenteric plexus and continuity with the rectum. They may contain ectopic tissue and malignant degeneration has been noted.

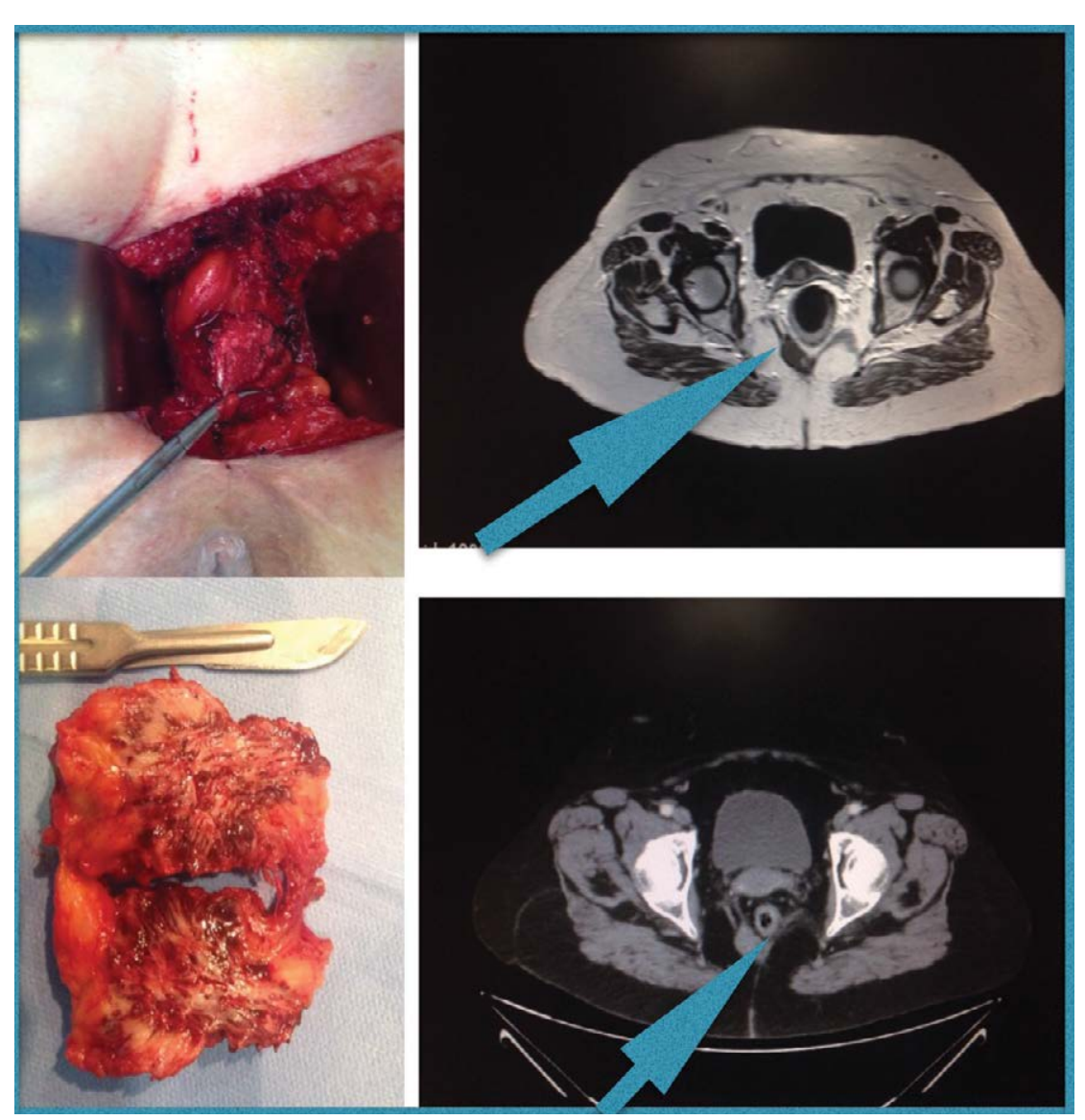

Figure $4 \mathrm{CT}$ and MRI illustrated a dermoid cyst. Specimen is showed. A tract of communication with rectum was doubled sutured. 


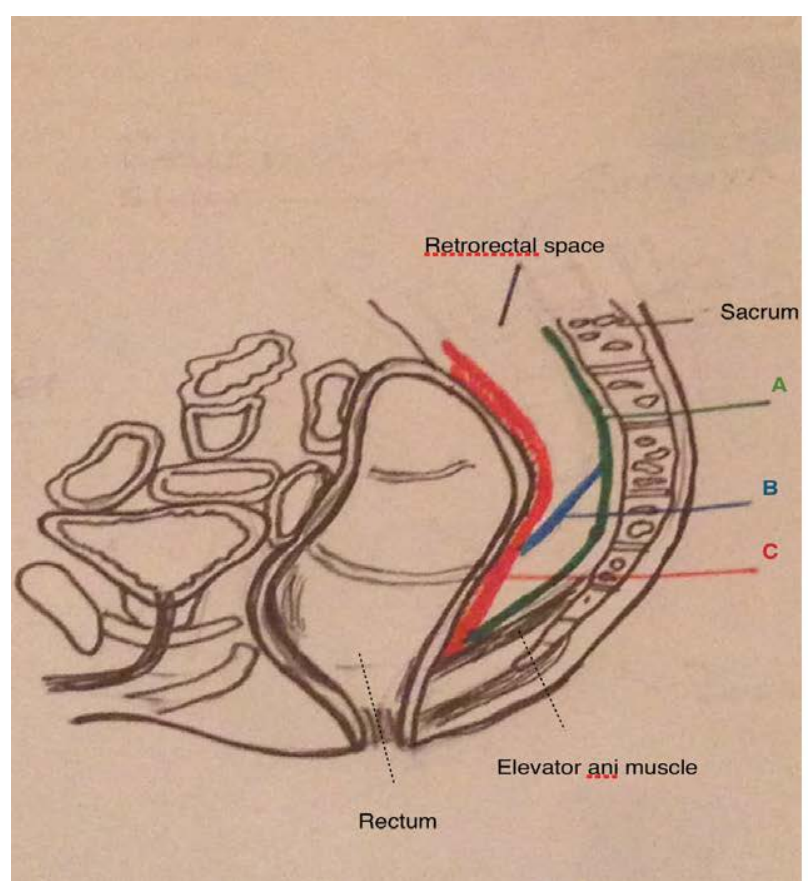

A

\section{Presacral fascia}

B

Waldeyer's fascia or retrorectal fascia divides the

rectrorectal space into superior and inferior compartment. It origins from pressacral fascia at S2-S4 level and passes caudally to fuse mesorectal fascia at $3-5 \mathrm{~cm}$ from anorectal junction.

It poses two leaves. Neither vascular structures nor nerves have been described between these two leaves.

Mesorectum and mesorectal fascia.

Figure 5 Anatomy of the presacral space is illustrated.

*Tailgut cysts: Also known as cystic hamartomas. They pose a columnar and transitional epithelium, and absence of welldefined muscular wall. Degeneration to adenocarcinoma has been reported.

*Teratomas: They are the most frequente presacral tumours in the infancy. They have malignant potential (risk of $10 \%$ to $40 \%)$. Cystic and solid appearance will be demonstrated in either CT or MRI.

Sacroccygeal chordomas: They are the most common malignat presacral tumours. They are solid tumours that grow slowly and invade adjacent tissues. Metastases to lungs, liver and bone have been reported and local recurrence rate is high even after resection. Anterior sacral meningocele: they can be presented with other congenital abnormalities such as bifid spine.

\section{Neurogenic tumours}

These account for $10 \%$ of all presacral tumours. They stem from peripheral nerves and most of them are benign: neurofibroma, neurilemoma and ganglioneuroma (85\%). Malignant tumours include neuroblastoma, ganglioneuroblastoma, ependymoma, and malignant peripheral nerve sheath tumours.

\section{Osseous tumours}

They originate in bone, cartilage, fibrous tissue and bone marrow. They have a slow growth and metastases in the lungs are associated to poor prognosis.

\section{Inflammatory tumours}

They have their origin in foreign bodies such barium, surgical sutures. etc

\section{Miscellaneous tumors}

Accounts for $1 \%$ to $25 \%$ of all presacral tumours and include: lymphomas, fibrosarcomas, liposarcomas, hystocitomas, lipomas, leiomyomas and hemagiomas.

Diagnosis can be delayed because most of patients will remain asymptomatic for years (26\% to $57 \%$ ) [5]. When symptoms appear they are varied and vague. Therefore, misdiagnosis and delayed diagnosis are not uncommon $[1,5,7,8]$. The presence of pain suggests either malignant process or infection in case of benign tumour. Other symptoms are constipation, paradoxical diarrhoea, headache (in case of meningocele), incontinence, dysuria etc.

In some occasions, they can be presented as recurrent perianal fistula, abscess or pilonidal cyst as a result of complication. It is important to remember this in case of recurrent perianal fistula or pilonidal cysts. According to some authors, malignancy is associated to pain and age over 60 years old. About gender, it is unclear. Jao reported a high incidence in males [3]. Conversely, Craig noted that malignancy occurred more frequently in women $[4,5,7,8,10]$. A comprehensive clinical assessment is mandatory to diagnose these lesions, and a rectal exam is essential. Rectal examination which includes digital rectal examination (palpation of a smooth posterior rectal wall and extramucosal mass) and rigid sigmoidoscopy are crucial not only for diagnosis but also for an adequate surgical approach [2,9]. Usually, tumours that are palpated in a digital rectal examination can be excised via posterior, and those who cannot be palpated are candidates to abdominal approach. Endoscopy must be performed to ensure that there is not transmural invasion of the tumour [2].

Moreover, transrectal ultrasound combined with rigid proctoscopy has a sensitivity of $100 \%$ [10]. 
Two important tools are MRI and CT, especially MRI that is considered a crucial element to decide the most optimal surgical procedure. Pelvic CT is useful to demonstrate bone invasion and destruction, nature of the lesion (solid or cystic) and location related to other structures.

However, pelvis MRI allows a better assessment of soft tissue, level of the lesion, anatomical boundaries of the tumour, extension and risk of malignancy. These data are crucial to reach an adequate surgical strategy. In addition, MRI is very sensitive in spinal imaging and pathology related to cord. Furthermore, chest and abdominal CT must be performed if malignancy is suspected $[1,2,4,5,7,8]$. The role of biopsy is controversial and motive of debate. We consider that preoperative biopsy should be performed only if it will provide information that may alter the management of the tumour such as to be candidate to neoadjuvant therapy (such as sarcomas) or to be treated only with chemotherapy (lymphomas). However, this seldom occurs. The main concern about biopsy is the complications that can follow: infection, hemorrhage, sepsis, meningitis, fistula, bowel perforation and risk of seeding through the biopsy tract $[4,5,6]$. Neither of our patients underwent preoperative biopsy, not even the third case that rejected surgery.

Finally, it is widely accepted that all retrorectal tumours, benign (symptomatic or asymptomatic) and malignant, must be excised as long as the patient is fit for surgery $[1,3-5,7,8,12]$.

Surgical strategy must be multidisciplinary and two questions must be considered: Circumferential resection or "enbloc" resection and posterior or abdominal approach?

*Abdominal approach: must be considered for lesions located above S3. The lesion must be separated from mesorectum and presacral fascia, and arterial supply ligated.

* Posterior approach: (Kraske procedure) is elected when lesions are located below the level of S3. Patient lies on Jackknife position. Midline incision or para sacral incision is performed. Ano -coccygeal ligament is excised, gluteus maximus muscles are separated laterally and S4-S5 can be excised in case of malignancy. Usually there is a plane between mesorectum and the lesion, especially in benign tumours. If a rectal wall defect occurs, it requires to be sutured in two layers. Malignant processes require excision in bloc. Duran tears must be identified and repaired to avoid cerebrospinal liquid leak or infection $[2,7,8]$.

*Combined approach: Abdominoperineal tumors between S3-S4 or involving of sacrum, pelvic viscera and pelvic side wall $[1,2,7]$. Patient is placed in a modified lateral position. Lower midline laparotomy is performed. Exploration of the cavity for metastases is important. Then sigmoid is mobilised and ureters identified. The lesion must be separated from mesorectum and presacral fascia to the level of elevators ani muscle. In case of malignat tumours with involvement of surrounding structures a resection in bloc is mandatory (low anterior rectum resection), and in some cases this will include sacrum and nerve roots
$[13,14]$. S3 root is required for urinary and fecal continence, so if S3 is excised, colostomy is necessary. Then abdomen is closed and patient placed in prono position, then posterior approach is performed in order to complete excision [1,2,9]. Recently, laparoscopic approach has been described to excision of these tumours [15]. Recurrence depends on nature of the tumour and quality of resection. Overall survival for benign tumours is approximately $100 \%$. However, malignant processes differ. Chordomas have a 10 year survival rate from $15 \%$ to $84 \%[2,3]$. The four patients reported on this paper were diagnosed with $\mathrm{MRI}$ or $\mathrm{CT}$ and rectoscopy. We also consider digital rectal examination essential as initial method of diagnosis, and it also provides relevant aspects about surgical approach. It is primordial to determine at what level the lesion is located. Our limit was S3, and surgery procedure was decided according to this limit. The most common lesion in our department was hamartoma and there was a tendency towards female gender. All the patients were treated by senior surgeons. Surveillance was performed with $\mathrm{MRI}$ and endoscopy. In one case, the patient rejected surgical treatment. We draw attention to the fact that this patient is under strict follow up with periodical MRI and endoscopies. We did not consider biopsy because findings on CT suggested a benign lesion. The lesion has remained stable for four years, and no other lesions have appeared. This supports the role of image techniques in determine the nature of the tumour. We recommend surveillance after surgery because of the incidence of recurrence reported. We perform a MRI at 6 months after surgery, and then annual. However, currently, consensus about surveillance lacks in the literature.

\section{Conclusion}

To summarise, the low incidence of these tumours and their vague symptomatology made them a challenge for the surgeon. Most of surgeons will only face one or two cases over their career.

Most of them are congenital and benign. An accurate diagnosis is crucial to achieve good results.

MRI, CT and endoscopy allow to plan an optimal treatment which involves surgery and in some case adjuvant treatment $[1,2,4,5,7,8,10]$. RM is an essential tool to determine the type of surgery: circumferential versus "en bloc" related to nature of tumour, posterior versus abdominal approach regarding relation to S3 $[6,8]$. Moreover, different specialists are required in order to excel optimal results. Biopsy is controversial. We highlight that preoperative biopsy is an unnecessary risk in most cases. However, it could be considered when neoadjuvant therapy is an option. Finally, surgery is considered the essential treatment for all patients fit for surgery. In addition, chemotherapy could be indicated in selected patients $[1,2,6,7,9,12]$.

\section{Acknowledges}

Thanks to Luciano Minaya Mateo for his help with the design of Figure 5. 


\section{References}

1 Jao SW, Beart RW, Spencer RJ, Reiman HM, Ilstrup DM (1985) Retrorectal tumors Mayo Clinic experience, 1960-1979. Dis Colon Rectum 28: 644-652.

2 Sagar AJ, Tan WS, Codd R, Fong SS, Sagar PM (2014) Surgical strategies in the management of recurrent retrorectal tumours. Tech Coloproctol 18: 1023-1027.

3 Hassan I, Wietfeldt ED (2009) Presacral tumors: Diagnosis and management. Clinics in Colon and Rectal Surgery 20: 84-93.

4 Sagar AJ, Koshy A, Hyland R, Rotimi O, Sagar PM (2014) Preoperative assessment of retrorectal tumours. Br J Surg 101: 573-577.

5 Messick CA, Hull T, Rosselli G, Kiran RP (2013) Lesions originating within the retrorectal space: a diverse group requiring individualized evaluation and surgery. J Gastrointest Surg 17: 2143-2152.

6 Chéreau N, Lefevre JH, Meurette G, Mourra N, Shields C, et al. (2013) Surgical resection of retrorectal tumours in adults: long-term results in 47 patients. Colorectal Dis 15: e476-e82.

7 Kildušis E, Samalavičius NE (2014) Surgical management of a retrorectal cystic hamartoma (tailgut cyst) using a trans-rectal approach: a case report and review of the literature. J Med Case 8-11.
8 Munteanu I, Badulescu A, Mastalier B, Munteanu ML, Diaconu E, et al. (2013) Retrorectal dermoid cyst: A rare clinical entity. Curr Health Sci J 39: 179-183.

9 Woodfield JC, Chalmers AG, Phillips N, Sagar PM (2008) Algorithms for the surgical management of retrorectal tumours. Br J Surg 95: 214-221.

10 Lin CZ, Jin K, Lan HR, Teng LD, Lin JJ, et al. (2011) Surgical management of retrorectal tumors: A retrospective study of a 9-year experience in a single institution. OncoTargets and Therapy 4: 203-208.

11 Neale JA (2011) Retrorectal tumours. Clin Colon Rectal Surg 24:149160.

12 Jin ZM, Peng JY, Zhu QC, Yin L (2011) Waldeyer's fascia: anatomical location and relationship to neighboring fasciae in retrorectal space. Surg Radiol Anat 33: 851-412.

13 Aranda-Narváez JM, González-Sánchez JA, Montiel-Casado C, Sánchez-Pérez B, Jiménez-Mazure C, et al. (2012) Posterior approach (Kraske procedure) for surgical treatment of presacral tumors World J Gastrointest Surg. 4: 126-130.

14 Imboden S, al-Fana A, Kuhn A, Mueller MD (2014) Pandora's box and retrorectal tumors in laparoscopy: A case report and review of the literature. Int J Surg Case Rep 5: 706-709. 